\title{
The Emerging Use of Smarthphone apps in Urology
}

\author{
Jonathan Makanjuola and Artaches Zakarian \\ King's College London, \\ United Kingdom
}

\section{Introduction}

A smartphone is defined as a phone enabled for internet or email use. Mobile technology presents an opportunity for urologists to continue to lead in development in medical technology Urology continues to be a technology-driven specialty with the advent of robotic surgical systems this has led to urologists becoming the world leaders in the use of such technology [1]. Applications (apps) are downloaded items of software onto a smartphone which fulfil a specific function or role. The worldwide market for smartphone applications has grown enormously in recent years. Revenues from applications in the first half of 2010 were estimated at $£ 1.4$ billion (\$2.2 billion), while in January 2012, Apple announced the twenty fifth billionth download from its App Store [2]. The Android market is the alternative to the Apple 'app store' developed by Google for Android OS devices [3]. Once downloaded, users can rate the app (out of 5 stars) on the app page so others can rate the usefulness of the app. Raters can also leave comment for prospective users to read.

\section{Emerging use of smartphone apps in urology}

Smartphones have the potential to improve diagnostic skills and education of a surgeon [4][5]. Urological apps available to download on the Apple App Store and Google Android Market are used for a wide variety of uses, including reference tools to aid diagnosis of urological conditions. Simple apps like 'Kidney 3D' a surgical anatomy tool and more sophisticated information apps like 'Prostate Cancer Calculator', a tool using a formula from Prostate Cancer Prevention trial to create a risk calculator designed to provide a preliminary assessment of risk of prostate cancer if a prostate biopsy is performed [6].

Urology apps have a broad range of uses like 'Advanced Urology' a urology reference app detaining the management of common urological conditions. There are many patient information apps like 'bladder pal' an app designed for patients to track fluid intake and urinary output. There are urological conference app like the 'SIU 2011 Berlin' app. It was created for attendees at the Société Internationale d'Urologie (SIU) conference where users can access the latest conference news, share contacts among the delegates; create a unique program for the conference based on the user's interests. 
There were 69 apps found following a key word search of the term 'Urology', 'Kidney', 'Bladder' and 'Prostate' to the search tab in the Apple App Store and The Android Market. Included were all of the apps that are based on urological disease for health professionals and patients. Excluded were apps with a focus on Nephrology and complementary or alternative treatments in urology. The earliest posted app at the time of our search was on 19th November 2009. There was a more than doubling of the number of urological apps found on the app sites between 2010 and 2011. The average cost of the urological apps was $£ 6.73 / \$ 10.64$; (range $£ / \$ 0$ - $£ 34.99 / \$ 55.82$ ), with 36 apps available for free. There were 52 apps produced by companies specialising in app development with 17 produced by Urologists. 35 apps were for reference, 18 for patient information, 6 for conference use, 3 for urology news and 2 for patient records in urological diseases. The types of applications are detailed in table $1.65 \%$ of the apps found were aimed at physicians, $33 \%$ aimed at patients and $2 \%$ at urology nurses.

\begin{tabular}{l|c} 
Type of app & Number \\
\hline Reference & 35 \\
Patient Information & 18 \\
Conference & 6 \\
Urology News & 3 \\
Patient Records & 2 \\
Decision support & 1 \\
Drawing tool & 1 \\
Logbook & 1 \\
Social Network & 1 \\
Urology Careers & 1
\end{tabular}

Table 1. The types of urological apps in the App Store and The Android Market.

There was an average 1 star rating of the entire urology apps reviewed. The app with the most ratings was Skyscape Medical Resources [7] with 696 raters and an average rating of 2.5 stars. There were a total of 8 comments posted about the urological apps (4 positive and 4 negative comments).

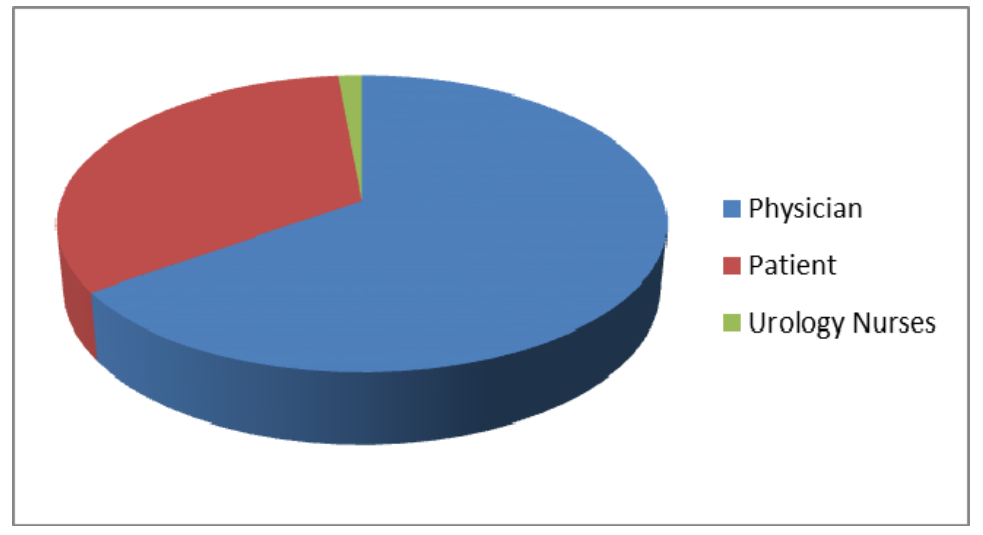

Fig. 1. The target user for urology apps in the App Store and The Android Market. 


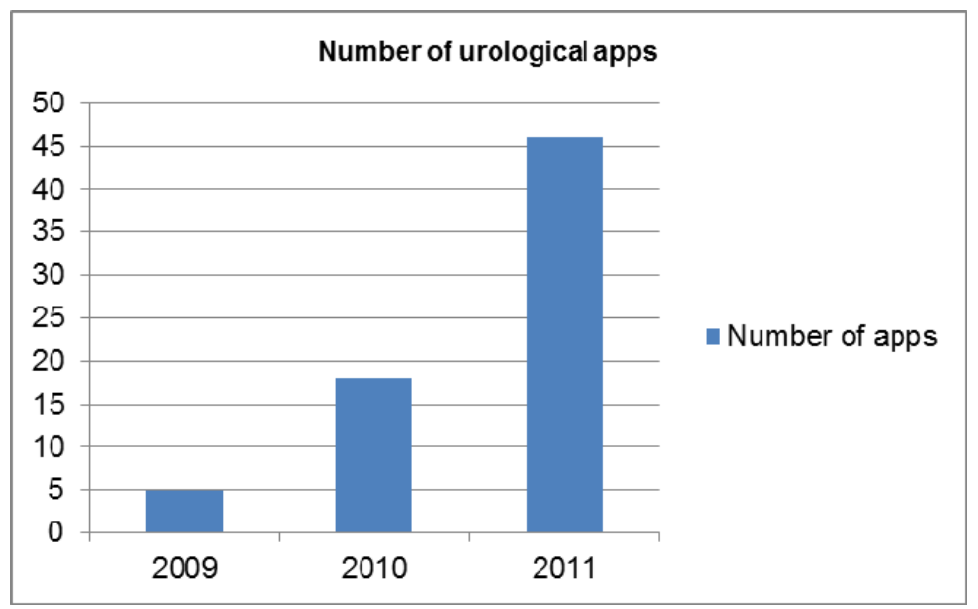

Fig. 2. The number of urology apps found in the App Store and The Android Market.

There is an exponential increase in the number of urology apps being produced each year. The use of these apps by urologists is difficult to determine by using the rating system provided by Apple and Android, as either they are not being downloaded or no rating is being left. As smartphones become an ever increasing part of modern life, healthcare is embracing this technological revolution. Urologists will be seeing patients who have read information on their urology apps, just like websites patients read before seeing their doctors in outpatient clinics. There are some negative aspects to the use of apps by patients and physicians. Regulation of these apps is lacking there is no regulation of the medical content. In July 2011, in an attempt to regulate medical apps the US Food and Drug Administration (FDA) are in public consultation regarding guidelines for the development a subset of mobile medical apps that may impact on the performance or functionality of currently regulated medical devices [8].

\section{Conclusion}

Apps provide an opportunity for urologists to engage with patients and offer a reference tool that can be accessed anywhere at any time on a smartphone. With this emerging market and increasing creation of medical apps, urologists in the future will be using more apps with focused urology content filtered to relevant and specific information. There will be an increase in patient information content in apps, presenting a unique challenge for urologists. This presents an opportunity for urological postgraduate bodies like the American Urological Association (AUA) or British Association of Urological Surgeons (BAUS) to lead the development of urological apps for use by physicians and patients.

\section{References}

[1] D Murphy, B Challacombe, M S Khan, and P Dasgupta. Robotic technology in urology. Postgrad Med J 2006;82:743-747

[2] Apple app store. Available at: www.apple.com. Accessed 02nd March 2012. 
[3] Google android Market. Available at: https://market.android.com/?hl=en. Accessed 16 $6^{\text {th }}$ October 2011.

[4] Dala-Ali BM, Lloyd MA, Al-Abed Y. The uses of the iPhone for surgeons. Surgeon. 2011 Feb;9(1):44-8. Epub 2010 Aug 21.

[5] Freshwater MF. iPhone and iPad applications for plastic surgeons.. J Plast Reconstr Aesthet Surg. 2011

[6] Thompson IM, Ankerst DP, Chi C, Goodman PJ, Tangen CM, Lucia MS, Feng Z, Parnes HL, Coltman CA Jr. Assessing prostate cancer risk: Results from the Prostate Cancer Prevention Trial J Natl Cancer Inst. 2006 Apr 19;98(8):529-34.

[7] Skyscape Inc. ${ }^{\circledR}$. Available at: http://www.skyscape.com/company/companyhome.aspx. Accessed $16^{\text {th }}$ October 2011.

[8] Food and Drug Administration. FDA Proposes Health 'App' Guidelines. http://www.fda.gov/ForConsumers/ConsumerUpdates/ucm263332.htm. Accessed 16 ${ }^{\text {th }}$ October 2011. 
(C) 2012 The Author(s). Licensee IntechOpen. This is an open access article distributed under the terms of the Creative Commons Attribution 3.0 License, which permits unrestricted use, distribution, and reproduction in any medium, provided the original work is properly cited. 